journal club

\title{
Auswirkungen hoher Dosen von Vitamin D auf die Immunzellpopulation
}

Fragestellung: Welche Auswirkungen hat eine Hochdosistherapie mit Cholecalciferol beziehungsweise Vitamin D auf die Immunzellpopulation von Patienten mit Multipler Sklerose (MS) und wie werden diese hohe Dosen vertragen?

Hintergrund: Die Rolle von Cholecalciferol in der Pathogenese der MS wird schon seit geraumer Zeit kontrovers diskutiert. Viele Studien belegen, dass Patienten mit einer MS deutlich erniedrigte Cholecaciferol-Spiegel im Serum aufweisen. Die eigentliche Tragweite dieser Beobachtungen kann bis heute aber nicht vollends interpretiert werden. Experimentell wurden im Tiermodell sowie in vitro zahlreiche immunmodulierende, antientzündliche und protektive Eigenschaften von Cholecalciferol gefunden und somit ein möglicher Einfluss auf den Krankheitsverlauf bei der MS postuliert.

Patienten und Methodik: Es handelt sich um eine randomisierte Singlecenterstudie mit Einschluss von 40 Patienten mit einer MS vom schubförmigen Verlaufstyp. Die Studienteilnehmer wurden in zwei Gruppen

Sotirchos ES, Bhargava P, Eckstein $C$ et al. Safety and immunologic effects of high- vs lowdose cholecalciferol in multiple sclerosis. Neurology 2016; 86: $382-90$ randomisiert, die täglich entweder 10.400 IU oder 800 IU Cholecalciferol für die Dauer von sechs Monaten erhielten. Die überwiegende Anzahl der Patienten wurde mit unterschiedlichen Basisthera- peutika oder mit Fingolimod oder Natalizumab behandelt, einer der Patienten erhielt Abatacept. Hauptgegenstand der Untersuchung war eine Veränderung der Immunzellpopulation mit Bestimmung der Interleukin $17^{+}-\mathrm{CD} 4^{+}-\mathrm{T}-Z$ Zellen, CD-161 $1^{+}-\mathrm{CD} 4^{+-}$ T-Zellen, Effektor- und Gedächtnis-T-Zellen sowie naiven CD4+-T-Zellen unter der Gabe von Cholecalciferol zum Zeitpunkt 0 (Baseline) sowie nach drei und nach sechs Studienmonaten.

Ergebnisse: Patienten aus der Hochdosisgruppe zeigten einen Anstieg des 25-Hydroxyvitamin-D-Spiegels um 34,9 ng/ml auf $59,7 \mathrm{ng} / \mathrm{ml}$, während in der Niedrigdosisgruppe ein Anstieg von $6,9 \mathrm{ng} / \mathrm{ml}$ auf $32,4 \mathrm{ng} / \mathrm{ml}$ zu verzeichnen war. Des Weiteren verringerte sich im Mittel der Anteil der Interleukin $17^{+}-\mathrm{CD} 4^{+}-\mathrm{T}-$ Zellen, der CD-161+-CD4+-T-Zellen und Effektor-GedächtnisT-Zellen in der Hochdosisgruppe, während der Anteil der zentralen Gedächtnis- sowie naiven $\mathrm{CD}^{+}$-T-Zellen leicht anstieg. In der Niedrigdosisgruppe zeigten sich keine signifikanten Veränderungen.

Schlussfolgerungen: Die Gabe von 10.400 IU Cholecalciferol reduziert die Frequenz von Interleukin $17^{+}-\mathrm{CD} 4^{+}-\mathrm{T}-Z$ Zellen. Hieraus folgern die Autoren, dass die antiinflammatorischen Eigenschaften von Cholecalciferol bei MS-Patienten möglicherweise über eine Reduktion dieser sogenannten TH17-Zellen vermittelt werden könnten. Weiterhin zeigte diese Untersuchung, dass hohe Vitamin-D-Dosen gut toleriert werden.

\section{- Kommentar von De-Hyung Lee, Erlangen}

\section{Die Bedeutung für den klinischen Alltag bleibt noch unklar}

Diese Studie könnte der Ausgangspunkt für weitergehende Untersuchungen sein, die sich verstärkt mit den immunmodulierenden Eigenschaften von Vitamin D bei MS-Patienten befassen. Die Beobachtung, dass in diesem Studienkollektiv die hochdosierte Gabe von 10.400 IU Cholecalciferol die Frequenz von sogenannten Th17-Zellen beeinflusst, ist äußerst interessant. Inwiefern die Ergebnisse allerdings direkt eine Modulation der MS-Pathogenese reflektieren, muss offen bleiben. Insbesondere die Bedeutung dieser Erkenntnisse für den klinischen Alltag bleibt unklar.

Dennoch bestärken die Erkenntnisse dieser Studie, dass Vitamin D zur multifaktoriellen Krankheitsentstehung beitragen könnte. Tatsächlich existiert eine Vielzahl von Untersuchungen, die eine Assoziation zwischen dem Vitamin-D-Haushalt und MS-Pathogenese vermuten. Limitierend wirken sich die kleine Gruppengröße und die heterogenen Begleittherapien aus, welche die Probanden erhalten haben. Hilfreich werden weitere prospektiv angelegten Studien mit einem größe- ren, gut standardisierten Patientenkollektiv sein. Die Ergebnisse der prospektiv angelegten placebokontrollierten Doppelblindstudie SOLAR, bei der die Wirksamkeit von Vitamin $D$ in Kombination mit einem beta-Interferon untersucht wurde, sollen demnächst veröffentlicht werden und können unter Umständen eine bessere Aussage über klinische Parameter bei MS-Patienten bringen.

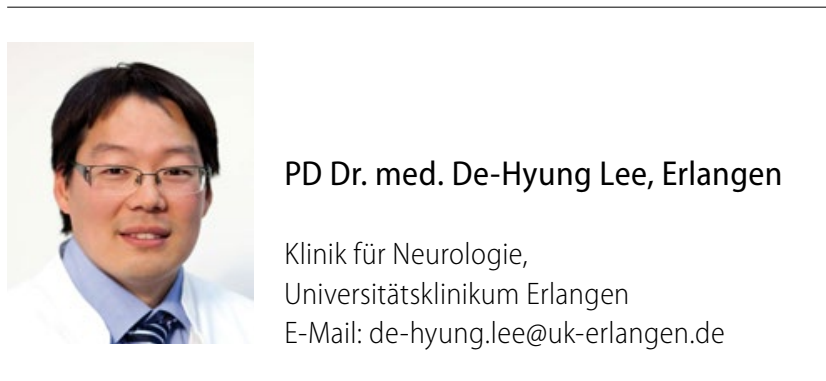

